\title{
Plane waves in quantum gravity: breakdown of the classical spacetime
}

\author{
Guillermo A. Mena Marugán \\ I.M.A.F.F., C.S.I.C., Serrano 121, 28006 Madrid, Spain \\ Manuel Montejo \\ Universitat de Barcelona, Diagonal 647, 08028 Barcelona, Spain
}

\begin{abstract}
Starting with the Hamiltonian formulation for spacetimes with two commuting spacelike Killing vectors, we construct a midisuperspace model for linearly polarized plane waves in vacuum gravity. This model has no constraints and its degrees of freedom can be interpreted as an infinite and continuous set of annihilation and creation like variables. We also consider a simplified version of the model, in which the number of modes is restricted to a discrete set. In both cases, the quantization is achieved by introducing a Fock representation. We find regularized operators to represent the metric and discuss whether the coherent states of the quantum theory are peaked around classical spacetimes. It is shown that, although the expectation value of the metric on Killing orbits coincides with a classical solution, its relative fluctuations become significant when one approaches a region where null geodesics are focused. In that region, the spacetimes described by coherent states fail to admit an approximate classical description. This result applies as well to the vacuum of the theory.
\end{abstract}

PACS number(s): 04.60.Ds, 04.30.-w

\section{INTRODUCTION}

The quantization of spacetimes that possess two commuting spacelike Killing vector fields has received considerable attention [1-7]. One of the main motivations for the study of this type of spacetimes is that they generally describe situations of interest in astrophysics and cosmology [8]. Actually, most of the families of spacetimes with two Killing vectors that have been quantized in the literature can be interpreted as gravitational waves that propagate either in Minkowski spacetime or in cosmological universes [1-4]. This is the case, e.g., of linearly polarized gravitational waves in cylindrically symmetric spacetimes, which were first analyzed quantum mechanically by Kuchař and Allen [5]. A consistent quantization of this gravitational system was achieved by Ashtekar and Pierri [1], whilst the most general model of cylindrical waves in vacuum gravity was quantized by Korotkin and Samtleben [2]. Preliminary discussions on the quantization of gravitational waves in spacetimes with planar symmetry can be found in Ref. [6]. A systematic analysis of purely gravitational plane waves in quantum geometrodynamics was recently carried out by the authors [3]. Finally, the quantization of the Gowdy cosmologies with the spatial topology of a three-torus was addressed in Refs. [1, 7. These Gowdy spacetimes can be thought of as inhomogeneous universes with compact sections of constant time that are filled with gravitational waves [9].

Another important motivation for the quantization of spacetimes with two commuting spacelike Killing vectors comes from their ability to provide a suitable arena where conceptual issues in quantum gravity can be advantageously discussed. To date, most of the gravitational systems that have been quantized to completion are min- isuperspace models [10]. Such gravitational systems are too simple to capture the quantum field structure of general relativity. In the presence of two commuting Killing vectors, however, Einstein gravity reduces to midisuperspace models, namely, gravitational models with an infinite number of degrees of freedom. It is a common belief that these models might mimic the complexity that should be present in a quantum field theory of general relativity.

In this context, the existence of quantum gravitational states that admit a classical description, or a semiclassical one if quantum matter is present [11, has been recently addressed [12,13]. By considering a model for linearly polarized gravitational waves with cylindrical symmetry, Ashtekar has discussed whether it is possible that quantum states are strongly peaked around classical spacetimes, assuming that the matter content is given by the expectation value of the energy-momentum tensor of the matter fields. After a dimensional reduction, cylindrical waves with linear polarization adopt the same formulation as axi-symmetric Einstein-Maxwell gravity in three dimensions [1, 12]. For the coherent states of the Maxwell field, it has then been proved that the quantum fluctuations in the three-dimensional metric are relatively small only if the coherent state contains neither too many photons nor photons of high frequency [12]. In particular, the three-dimensional metric of the ground state is strongly peaked around Minkowski spacetime. At least from this three-dimensional point of view, one can say that the spacetime foam around the vacuum is smooth. In addition, it has been shown that, although one can construct states that diminish the uncertainty in the three-dimensional metric, they induce a loss of coherence in the Maxwell field [14]. 
The above considerations seem to indicate that, in a certain sector of quantum gravity, large quantum effects may preclude an approximate classical description of the spacetime. However, several points remain obscure in this tentative conclusion. On the one hand, the discussion in Refs. 12,14 has been carried out from a three-dimensional perspective. Although axi-symmetric Einstein-Maxwell theory in three dimensions is equivalent to four-dimensional, cylindrical gravitational waves with linear polarization, some issues concerning the interpretation of physical quantities (e.g., the metric) may depend on the particular approach adopted. On the other hand, the results obtained could be an artifact of the particular system studied. In order to discuss the relevance of these results, one would like to analyze the classical limit of other midisuperspace models. Actually, large gravitational fluctuations similar to those described by Ashtekar [12] have also been found (again from a threedimensional perspective) in a model with toroidal symmetry 15]. In the present work, we will study the quantum behavior of the four-dimensional metric in another family of spacetimes with two commuting Killing vectors, namely, the model for linearly polarized plane waves in vacuum gravity that was discussed in Ref. [3]. The analysis of the classical limit for plane waves is particularly interesting, because these spacetimes show the remarkable feature of focusing the null cones 16 . In the neighborhood of the points where null cones are focused, one should expect that quantum gravity effects could be especially important.

The rest of the paper is organized as follows. We first present our midisuperspace model in Sec. II, where we briefly summarize the reduction of source-free Einstein gravity carried out in Ref. [3] for the case of linearly polarized plane waves. The degrees of freedom of this model are given by a metric function $Y$ that, on classical solutions, depends only on one of the spacetime coordinates, $u$. In Sec. III we show that, when the field $Y$ corresponds to a flat solution outside a bounded, fixed interval for the coordinate $u$, it can be described in terms of an infinite number of discrete modes. The most general case in which the spacetime is not restricted to be flat in any region is studied in Sec. IV. The field $Y$ can then be expanded in a continuous set of modes. The quantization of these two models, with either discrete or continuous modes, is discussed in Sec. V. In particular, we introduce regularized operators that represent the metric functions. In Sec. VI we define coherent states for the quantum operator associated with the field $Y$. At least on the orbits of the two spacelike Killing vectors of the model, the expectation value of the metric in any coherent state turns out to coincide with a classical plane-wave solution. We then study the fluctuations in the metric on such solutions and prove that they become large when one approaches a region where null cones are focused. As a consequence, the classical description of the spacetime breaks down in the vicinity of that region for all coherent states, including the vacuum. Finally, we discuss our results in Sec. VII.

\section{LINEARLY POLARIZED PLANE WAVES}

Purely gravitational plane waves are vacuum solutions to the Einstein equations that are characterized by possessing as much symmetry as do plane electromagnetic waves in flat spacetime, namely, a five dimensional group of motions [17]. These spacetimes are a particular type of plane-fronted gravitational waves with parallel rays (ppwaves 18 ) and were first considered by Baldwin and Jeffrey [19. One can interpret these waves as describing the gravitational field produced by a radiating body at great distances 18,19].

Although there exists a system of coordinates, called harmonics, which allows to cover each of the spacetimes for plane waves with a single chart, the symmetries of the wave are much more clearly displayed by using group coordinates 8,18 . The metric can then be written

$$
d s^{2}=-d U d V+h_{a b}(U) d x^{a} d x^{b} .
$$

Here, $a, b=1,2$ and the coordinates $x^{a}$ and $V$ run over the real line. The coordinate $U$, on the other hand, has a restricted domain of definition. It is possible to show that, as $U$ decreases (with a suitable choice of orientation) from any fixed initial value, a point is reached where the determinant of the two-metric $h_{a b}$ vanishes [8] (except in purely Minkowski spacetime). This point $U_{f}$ is a coordinate singularity. As a consequence, group coordinates cannot be globally employed to describe the whole of the spacetime. The existence of this coordinate singularity is intimately related to the focusing effect produced by plane waves [16]. Actually, one can prove that null cones are focused on the hypersurface $U=U_{f}$.

If the metric function $h_{12}$ vanish, the plane wave is said to be linearly polarized [18]. We will restrict our discussion to this subfamily of plane waves from now on.

In addition, it can be seen that, modulo a reversal of the coordinates $U$ and $V$ and a scale transformation of the form $x^{a} \rightarrow A x^{a}$, with $A$ a constant, every gravitational plane wave presents a region where the determinant of the two-metric $h_{a b}$ increases with $U$ from zero to the unity [3]. In that region, one can perform a change of coordinates from $U$ to a new coordinate that, in principle, runs over the whole real axis:

$$
u=-\ln \left(-\frac{1}{2} \ln \left[\operatorname{det} h_{a b}(U)\right]\right) .
$$

The metric in the considered region adopts then the expression [3]

$$
\begin{aligned}
d s^{2} & =-z_{0}^{\prime}(u) e^{z_{0}(u) / 2} e^{\Phi(u)} d u d V \\
& +e^{z_{0}(u)}\left[e^{-y(u)}\left(d x^{1}\right)^{2}+e^{y(u)}\left(d x^{2}\right)^{2}\right],
\end{aligned}
$$

with 


$$
\Phi(u)=\int_{u_{c}}^{u} \frac{d r}{2 z_{0}^{\prime}(r)}\left[y^{\prime}(r)\right]^{2}, \quad z_{0}(u)=-e^{-u} .
$$

Here, $z_{0}^{\prime}(u)$ and $y^{\prime}(u)$ are the derivatives of $z_{0}(u)$ and $y(u)$, respectively, and $u_{c}$ is a constant. In terms of $u$, the coordinate singularity that reflects the focusing effect exerted by the wave has been driven to minus infinity. Notice also that, from Eqs. (2.1) and (2.3), the explicit expression of the group coordinate $U$ as a function of $u$ can be obtained, instead of by inverting relation (2.2), by integrating the equation

$$
d U=z_{0}^{\prime}(u) e^{z_{0}(u) / 2} e^{\Phi(u)} d u .
$$

The metric given above is a solution to the vacuum Einstein equations for any choice of the arbitrary function $y$. It describes the most general gravitational plane wave with linear polarization, except in that it does not represent the whole of the spacetime that can be covered with harmonic coordinates, but only a part of it. Apart from neatly displaying the symmetries of plane waves, the system of coordinates adopted has an important advantage. After the change $V=2 t-u$, metric (2.3) can be interpreted by its own as corresponding to a globally hyperbolic spacetime which possesses two commuting spacelike Killing vector fields, namely, $\partial_{x^{a}}$. Therefore, in order to analyze the quantization of our gravitational system, one can start with the Hamiltonian formulation of general relativity for spacetimes with two commuting Killing vectors. Moreover, for this kind of spacetimes one can consistently restrict all considerations to the case that the surface with coordinates $t$ and $u$ is orthogonal to the group orbits spanned by the Killing vectors, as it happens for plane waves. This orthogonality condition removes all the gauge freedom related to diffeomorphisms of the coordinates $x^{a}[3]$.

To eliminate all the non-physical degrees of freedom and arrive at a midisuperspace model that describes only linearly polarized plane waves in source-free gravity, one must introduce additional gauge-fixing and symmetry conditions, as it is explained in Ref. [3]. The reduced system that one attains in this way is totally free of constraints, has vanishing reduced Hamiltonian [3] and its only degrees of freedom are given by a single field $Y(u)$. In principle, this field may also depend on the time coordinate $t$; however, since the dynamical evolution of our reduced model is trivial in the system of coordinates adopted, $Y$ remains time independent for all classical solutions. The metric of the model reproduces expression (2.3) after the replacements $V=2 t-u$ and

$$
y(u)=\sqrt{2} e^{-z_{0}(u) / 2} Y(u) .
$$

Hence, the (time independent) classical solutions of our midisuperspace model are precisely the purely gravitational plane waves with linear polarization. On the other hand, the reduced action of the system and the symplectic structure on any section of constant time are [3]

$$
S=\int_{t_{0}}^{t_{f}} d t \int_{\Sigma} d u Y^{\prime} \dot{Y}, \quad \Omega=\int_{\Sigma} d u \mathbf{d} Y^{\prime} \wedge \mathbf{d} Y .
$$

Here, $t_{0}$ and $t_{f}$ denote the initial and final values of the time coordinate, the prime stands for the derivative with respect to the coordinate $u$, and the dot represents the time derivative (which vanishes only on classical solutions). In these expressions, surface contributions to the action coming from sections of constant time have been neglected, and an overall constant factor has been set equal to the unity by a suitable choice of the (effective) Newton constant [3]. In addition, $\Sigma$ denotes the domain of definition of the coordinate $u$. In principle, this domain is the real axis. Nevertheless, it is easy to check that the reduction process performed in Ref. [3] can be straightforwardly generalized to the case that $\Sigma$ is any fixed interval of the real line.

\section{A MODEL WITH DISCRETE MODES}

Let us now analyze the case in which the spacetime is flat outside a fixed region $u \in I_{L} \equiv[-L, L]$, where $L$ is a positive constant. To be more precise, we assume that the function $y(u)$ of the line element (2.3) takes on constant values in the region $u>L$ and in $u<-L$. As a consequence, the same must happen with the metric function $\Phi(u)$ in Eq. (2.4). One can then easily check that, for $|u|>L$, the spacetime is flat [17]. Inside the interval $I_{L}$, on the other hand, the function $y(u)$ [and hence the field $Y(u)$ ] are left arbitrary. The metrics considered can be interpreted as describing a kind of sandwich waves 17. For all practical purposes, one can then obviate the flat regions $|u|>L$ and replace $\Sigma$ with $I_{L}$ in the expressions of the reduced action and symplectic structure (2.7).

We will further assume that the plane-wave solutions considered are sufficiently smooth. In particular, the restriction of $Y(u)$ to the interval $I_{L}$ is continuous. The field $Y$ has then a well-defined limit as $u$ approaches the endpoints of this interval from its interior (namely, when $u$ tends to $L^{-}$and $-L^{+}$). The following argument shows that one can then restrict the discussion to solutions that satisfy:

$$
\lim _{u \rightarrow L^{-}} Y(u)=\lim _{u \rightarrow-L^{+}} Y(u) .
$$

Suppose that the field $Y(u)$ does not verify this condition. By adding to $Y$ a suitable function of the form $C e^{z_{0}(u) / 2}$, where $C$ is a real constant, we can always obtain a new field $\bar{Y}(u)$ that satisfies Eq. (3.1). Moreover, it is easily checked that the plane-wave metrics obtained with the fields $Y(u)$ and $\bar{Y}(u)$ differ only by the scale transformation $x^{1} \rightarrow A x^{1}$ and $x^{2} \rightarrow x^{2} / A$, where the constant $A$ equals $e^{-C / \sqrt{2}}$. Since this scale transformation leaves invariant the domains of definition of the coordinates $x^{a}$, which are the real line, the geometries described by the 
fields $Y(u)$ and $\bar{Y}(u)$ can be considered equivalent. The boundary condition (3.1) removes then the corresponding overcounting of equivalent geometries.

Condition (3.1) allows us to extend the field $Y(u)$, restricted to $I_{L}$, to a periodic function over the entire real line, its period being equal to $2 L$. Since this periodic function coincides with our field in the whole interval of interest, $I_{L}$, we will denote it also by $Y(u)$, to keep the notation as simple as possible. The periodicity (and smoothness) of $Y(u)$ guarantees that it can be expanded in the following Fourier series:

$$
Y(u)=a_{0}+\sum_{n=1}^{\infty} \frac{1}{2 \sqrt{\pi n}}\left(a_{n} e^{-i n \pi u / L}+a_{n}^{\star} e^{i n \pi u / L}\right) .
$$

In order for $Y(u)$ to be real, $a_{n}^{\star}$ must be the complex conjugate of $a_{n}$, and $a_{0}$ must be real. The Fourier coefficients in this expression may, in principle, depend on the coordinate $t$, although they become time independent on classical solutions (recall that the reduced Hamiltonian vanishes).

Employing condition (3.1) and Eq. (2.7), it is not difficult to show that the Fourier coefficient $a_{0}$ disappears from the expressions of the reduced action and symplectic structure of the model. Therefore, the zero mode decouples from the other degrees of freedom of the system. In particular, this implies that we can consistently restrict all considerations to the case in which $a_{0}$ takes a specific value, independent of the coordinate $u$. We can take advantage of this fact to demand that the field $Y$ vanishes at a certain point $u_{0}$ of the interval $I_{L}$. In other words, we can set $a_{0}=-Y\left(u_{0}\right)$. Note that, with this choice, we are selecting the value of $Y$ at $u_{0}$ as the reference value with respect to which the field $Y$ is going to be measured. The Fourier series (3.2) becomes then

$$
\begin{aligned}
Y\left(u \mid u_{0}\right) & =\sum_{n=1}^{\infty} \frac{1}{2 \sqrt{\pi n}}\left[a_{n}\left(e^{-i n \pi u / L}-e^{-i n \pi u_{0} / L}\right)\right. \\
& \left.+a_{n}^{\star}\left(e^{i n \pi u / L}-e^{i n \pi u_{0} / L}\right)\right],
\end{aligned}
$$

which can be interpreted as a bilocal field (i.e., the difference between the values of a field at two points). In the above equation, we have made explicit the dependence on the point $u_{0}$. On the other hand, substituting the above series in the reduced symplectic structure and recalling that the zero mode decouples from the system, we obtain $\Omega=-i \sum_{n=1}^{\infty} \mathbf{d} a_{n} \wedge \mathbf{d} a_{n}^{\star}$. Hence, the only non-vanishing Poisson brackets between the Fourier coefficients are $\left\{a_{n}, a_{m}^{\star}\right\}=-i \delta_{n}^{m}$, where $\delta$ denotes the Kronecker delta. Since the coefficients $a_{n}(n \geq 1)$ are generally complex and conjugate to $a_{n}^{\star}$, the degrees of freedom of our model can then be interpreted as an infinite and discrete set of annihilation and creation like variables.

The quantization of the model can be achieved by introducing a Fock representation, as we discuss in Sec. V.
In order to avoid ultraviolet divergences in that quantization, the operator that represents the field $Y$ needs to be regularized. From a physical point of view, such a regularization can be justified as follows. In any real measurement of the (bilocal) field $Y\left(u \mid u_{0}\right)$, the positions of the points $u$ and $u_{0}$ will not be determined with total accuracy. One rather expects that the measurement will be performed over a small neighborhood of each of these points; the result will be an average of the form

$$
Y_{R}\left(u \mid u_{0}\right)=\int_{\mathbb{R}} d \bar{u} g(u-\bar{u}) \int_{\mathbb{R}} d \bar{u}_{0} g\left(u_{0}-\bar{u}_{0}\right) Y\left(\bar{u} \mid \bar{u}_{0}\right),
$$

where $g(u)$ is a smooth $\left[C^{\infty}(\mathbb{R})\right]$ test function of small compact support and unit integral, $\int_{\mathbb{R}} d u g(u)=1$.

Some comments are in order, concerning this formula. Since the original field $Y$ (which is the physically relevant one) coincides with its periodic extension only in the interval $I_{L}$, the same applies to the bilocal field $Y\left(u \mid u_{0}\right)$ with respect to its two arguments. As a consequence, the bilocal field in expression (3.4) can be substituted by the Fourier series (3.3) only if the integrals in that expression can be restricted to the interval $I_{L}$. Let then $I_{\epsilon} \equiv[-\epsilon, \epsilon]$ be the support of $g$, where, according to our previous discussion, $\epsilon \ll L$. This last condition guarantees that our measurements are good enough as to differentiate a huge number of regions in $I_{L}$. It is then easy to check that, as far as the points $u$ and $u_{0}$ belong to the interval $I_{L-\epsilon} \equiv[-L+\epsilon, L-\epsilon]$, the integrals in Eq. (3.4) do not receive contributions from regions outside $I_{L}$.

Substituting then the Fourier expansion of $Y\left(u \mid u_{0}\right)$ and recalling the properties of the function $g$, one obtains

$$
\begin{gathered}
Y_{R}\left(u \mid u_{0}\right)=\frac{1}{\sqrt{2}} \sum_{n=1}^{\infty}\left[f_{n}^{\star}\left(u \mid u_{0}\right) a_{n}+f_{n}\left(u \mid u_{0}\right) a_{n}^{\star}\right], \\
f_{n}\left(u \mid u_{0}\right)=\frac{e^{i n \pi u / L}-e^{i n \pi u_{0} / L}}{\sqrt{n}} \tilde{g}\left(\frac{n \pi}{L}\right),
\end{gathered}
$$

$f_{n}^{\star}$ being the complex conjugate of $f_{n}$ and $\tilde{g}$ denoting the Fourier transform of $g$ [20]. For points $u$ or $u_{0}$ whose absolute value lies between $L-\epsilon$ and $L$, Eqs. (3.5) and (3.6) must be modified. In the following, however, we will only consider the case $u, u_{0} \in I_{L-\epsilon}$, i.e., we will assume that all measurements of the field are made in the interior of the interval $I_{L}$ and sufficiently far from its endpoints.

On the other hand, since $g(u)$ is a smooth function of compact support, its Fourier transform belongs to $\mathcal{S}(\mathbb{R})$, the Schwartz space of smooth test functions of rapid decrease [20]. Using this property, it is not difficult to prove that the sequence $f \equiv\left\{f_{n} ; n \geq 1\right\}$ is square summable, $f \in l^{2}$. This fact will be employed in Sec. V to introduce a well-defined operator that represents the measured field $Y_{R}\left(u \mid u_{0}\right)$. Let us finally comment that, if we had not performed an average over the positions of $u$ and $u_{0}$, expressions (3.5) and (3.6) would still have been valid for the bilocal field $Y\left(u \mid u_{0}\right)$, but with the function $\tilde{g}$ replaced 
with the unit function divided by $\sqrt{2 \pi}$. In that case, the resulting sequence $f$ would have not belonged to the Hilbert space $l^{2}$, owing to the divergent contribution of the high-frequency modes $(n \gg 1)$.

\section{CONTINUOUS MODE EXPANSION}

We now return to the general case in which the field $Y(u)$ describes an arbitrary plane wave with linear polarization. As a boundary condition, we will demand the field to be of order unity at plus and minus infinity (we will say that a function $f(u)$ is of order $g(u)$ at $u_{0}$, and write $f(u)=O[g(u)]$ as $u \rightarrow u_{0}$, if the limit of $f / g$ exists at $\left.u_{0}\right)$. In this situation, similar arguments to those presented for the model with discrete modes show that, in order to avoid overcounting of equivalent geometries, one can restrict all considerations to fields that satisfy

$$
\lim _{u \rightarrow \infty} Y(u)=\lim _{u \rightarrow-\infty} Y(u) .
$$

Let us next extract from $Y$ its value at infinity. This value can be regarded as a function of the coordinate $t$ that, like the field $Y$, remains constant on classical solutions. Employing condition (4.1), one can check that this function has vanishing contribution to the reduced action and symplectic structure of the system, as it happened with the Fourier coefficient $a_{0}$ in the model of Sec. III. Thanks to this decoupling, one can consistently analyze any sector of the space of solutions where the considered function takes a specific value. We will benefit from this fact and limit our discussion to fields $Y\left(u \mid u_{0}\right)$ that vanish at a given point, $u_{0}$, as we did in the previous section. This restriction can again be interpreted as the choice of a reference value for the field $Y$.

We will finally assume that, apart from its constant value at infinity, the field $Y$ can be expressed as a Fourier transform. This assumption is the analogue of the expansion of $Y$ in a Fourier series employed in Sec. III. Recalling the restriction to fields that vanish at $u_{0}$, we can then write

$$
\begin{aligned}
Y\left(u \mid u_{0}\right) & =\frac{1}{2 \sqrt{\pi}} \int_{0}^{\infty} \frac{d k}{\sqrt{k}}\left[a(k)\left(e^{-i k u}-e^{-i k u_{0}}\right)\right. \\
& \left.+a^{\star}(k)\left(e^{i k u}-e^{i k u_{0}}\right)\right] .
\end{aligned}
$$

Since $Y\left(u \mid u_{0}\right)$ is real, the functions $a(k)$ and $a^{\star}(k)$ must be complex conjugate to each other. On the other hand, the boundary condition (4.1) can now be translated into conditions on the function $a(k)$. Let us define

$$
b(k)=\theta(k) a(k)+\theta(-k) a^{\star}(|k|), \quad c(k)=\frac{b(k)}{\sqrt{2|k|}},
$$

where $\theta$ is the Heaviside step function. It is then easy to check that $Y\left(u \mid u_{0}\right)$ equals $\tilde{c}(u)-\tilde{c}\left(u_{0}\right)$, where $\tilde{c}$ is the Fourier transform of $c$. Therefore, it suffices that $c$ is absolutely integrable $\left[c \in L^{1}(\mathbb{R})\right]$ to guarantee that condition (4.1) is fulfilled, because in that case $\tilde{c}$ is a continuous function that vanishes at infinity 20]. In particular, one can prove that $c$ belongs to $L^{1}(\mathbb{R})$ provided that $a(k)$ is square integrable over the positive real axis $\left[a \in L^{2}\left(\mathbb{R}^{+}\right)\right]$and there exist constants $\alpha>1 / 2$ and $\beta<1 / 2$ such that

$$
a(k)=O\left[k^{-\alpha}\right] \text { as } k \rightarrow \infty, \quad a(k)=O\left[k^{-\beta}\right] \text { as } k \rightarrow 0 .
$$

All these requirements are satisfied, e.g., if the function $b(k)$, defined above, belongs to the Schwartz space $\mathcal{S}(\mathbb{R})$.

We can regard the complex conjugate functions $a(k)$ and $a^{\star}(k)$ as the degrees of freedom of our model. In principle, these functions might depend on the time coordinate $t$; however, since the field $Y$ remains constant on classical solutions, they are classically time independent. In addition, replacing expression (4.2) in the symplectic structure [and recalling condition (4.1)], one obtains

$$
\Omega=-i \int_{0}^{\infty} d k \mathbf{d} a(k) \wedge \mathbf{d} a^{\star}(k),
$$

so that the only non-vanishing Poisson brackets between our variables are $\left\{a(k), a^{\star}(\bar{k})\right\}=-i \delta(k-\bar{k})$. The degrees of freedom for linearly polarized plane waves can thus be interpreted as a continuous set of annihilation and creation like variables, whose quantization can be carried out by introducing a Fock representation.

As we argued in Sec. III, any physical measurement of the (bilocal) field $Y\left(u \mid u_{0}\right)$ would imply an average of the form (3.4) over the positions of $u$ and $u_{0}$. Employing Eq. (4.2), this average leads to

$$
Y_{R}\left(u \mid u_{0}\right)=\int_{0}^{\infty} \frac{d k}{\sqrt{2}}\left[f^{\star}\left(k, u \mid u_{0}\right) a(k)+f\left(k, u \mid u_{0}\right) a^{\star}(k)\right],
$$

where, for $k>0$,

$$
f\left(k, u \mid u_{0}\right)=\frac{e^{i k u}-e^{i k u_{0}}}{\sqrt{k}} \tilde{g}(k) .
$$

It is worth noting that the above expressions can be obtained from Eqs. (3.5) and (3.6) in the continuum limit (when $L$ and $n$ tend to infinity, keeping $k=n \pi / L$ finite), a fact that supports our conclusions. Notice also that, in the limit $L \rightarrow \infty$, the region where the spacetime is flat for the model of Sec. III is driven to infinity, whereas the interval $I_{L-\epsilon}$ [namely, the region where formulas (3.5) and (3.6) are valid] becomes the real line. On the other hand, since $g$ is a smooth test function of compact support, its Fourier transform $\tilde{g}$ belongs to the Schwartz space $\mathcal{S}(\mathbb{R})$. It then follows that $f\left(k, u \mid u_{0}\right)$ is a square integrable function over the positive real line for all finite values of $u$ and $u_{0}$. This property will be used in the next section to attain a well-defined operator that represents the field $Y_{R}$ quantum mechanically.

To close this section, let us comment that, under the change of coordinates $u=x+t$, the field $Y\left(u \mid u_{0}\right)$ can 
actually be interpreted as a bilocal field constructed from the left-mover part of a massless scalar field in two dimensions (those corresponding to the coordinates $x$ and t) 21]. It is well known that such a scalar field presents infrared and ultraviolet divergences [21]. In our model, however, the infrared divergences have been eliminated by considering bilocal fields, whilst the average over positions has taken care of the ultraviolet divergences. This explains why the function $f\left(k, u \mid u_{0}\right)$ is square integrable: the convergence near $k=0$ has been achieved by subtracting contributions from the point $u_{0}$, whereas the convergence at infinity is ensured by the decay of $\tilde{g}$.

\section{QUANTUM THEORY}

We have argued that, for the two models considered in this work, a measurement of the field $Y$ (assumed to vanish at the point $u_{0}$ ) should lead to a result of the form:

$$
Y_{R}\left(u \mid u_{0}\right)=\frac{1}{\sqrt{2}}\left[<a, f\left(u \mid u_{0}\right)>+<a^{\star}, f^{\star}\left(u \mid u_{0}\right)>\right] .
$$

Here, $f$ and $f^{\star}$ are two vectors of a complex Hilbert space, $\mathcal{H}$, which are complex conjugate to each other and depend on the points $u$ and $u_{0}$. In addition, $<,>$ denotes the inner product on $\mathcal{H}$, defined as an antilinear mapping of its second argument. For the discrete model analyzed in Sec. III, $\mathcal{H}$ is the Hilbert space $l^{2}$ of square summable sequences, $f\left(u \mid u_{0}\right)$ denotes the sequence $\left\{f_{n}\left(u \mid u_{0}\right) ; n \geq 1\right\}$ given in Eq. (3.6), and $a$ and $a^{\star}$ stand for the discrete set of annihilation and creation like variables $\left\{a_{n} ; n \geq 1\right\}$ and $\left\{a_{n}^{\star} ; n \geq 1\right\}$, respectively. For the general case of linearly polarized plane waves, on the other hand, $\mathcal{H}$ is the Hilbert space $L^{2}\left(\mathbb{R}^{+}\right), f\left(u \mid u_{0}\right)$ is the function of $k \in \mathbb{R}^{+}$defined in Eq. (4.6), and $a$ and $a^{\star}$ denote the continuous set of annihilation and creation like variables $a(k)$ and $a^{\star}(k)$.

The quantization of the (bilocal) field $Y_{R}\left(u \mid u_{0}\right)$ can then be achieved by constructing a Fock representation of the annihilation and creation like variables. We will call $\mathcal{H}^{(n)}$ the tensor product $\otimes_{m=1}^{n} \mathcal{H}$. Let also $S_{n}$ be the symmetrization operator defined on $\mathcal{H}^{(n)}$ by its action on states of the form $\phi^{(n)} \equiv \phi_{1} \otimes \cdots \otimes \phi_{n}$ :

$$
S_{n} \phi^{(n)}=\sum_{\sigma} \frac{1}{n !} \phi_{\sigma(1)} \otimes \cdots \otimes \phi_{\sigma(n)} \equiv \phi_{s}^{(n)},
$$

where the summation is over all permutations $\sigma$. The symmetric Fock space over $\mathcal{H}$ is $\mathcal{F}_{s}(\mathcal{H})=\oplus_{n=0}^{\infty} \mathcal{H}_{s}^{(n)}$, where $\mathcal{H}_{s}^{(n)}=S_{n} \mathcal{H}^{(n)}$ is called the $n$-th particle subspace 20]. Let finally $\mathcal{F}_{0} \subset \mathcal{F}_{s}(\mathcal{H})$ be the dense subspace of finite particle vectors, i.e., the subspace of vectors $\phi_{s} \equiv\left\{\phi_{s}^{(n)} ; n \geq 0\right\}$ such that $\phi_{s}^{(n)}$ vanishes for all but a finite set of indices $n$. We can then define an annihilation operator $\hat{a}(f)$ on $\mathcal{F}_{s}(\mathcal{H})$, with domain $\mathcal{F}_{0}$, via its action on vectors of the type (5.2):

$$
\hat{a}(f) \phi_{s}^{(n)}=\sum_{\sigma} \frac{\sqrt{n}}{n !}<\phi_{\sigma(1)}, f>\phi_{\sigma(2)} \otimes \cdots \otimes \phi_{\sigma(n)}
$$

with $n \geq 1$, whereas $\hat{a}(f)$ vanishes on $\mathcal{H}_{s}^{(0)}$ [20]. This operator represents the classical quantity $\langle a, f\rangle$ and is closable for any $f \in \mathcal{H}$. Its adjoint is the creation operator $\hat{a}^{\star}(f)$, which represents the variable $\left\langle a^{\star}, f^{\star}\right\rangle$. Its restriction to the subspace of finite particle vectors can be obtained from the formula 20]:

$$
\hat{a}^{\star}(f) \phi_{s}^{(n)}=\sqrt{n+1} S_{n+1}\left(f \otimes \phi_{s}^{(n)}\right) .
$$

Notice that, from our definitions, $\hat{a}^{\star}(f)$ is linear in $f$, whereas $\hat{a}(f)$ is antilinear.

It is possible to understand each vector $\phi_{s}^{(n)}$ in $\mathcal{H}_{s}^{(n)}$ as a quantum state $\mid \phi_{s}^{(n)}>$ obtained from a vacuum $\mid 0>$ by the action of $n$ creation operators. For vectors given by formula (5.2), e.g., one has:

$$
\left|\phi_{s}^{(n)}>=\frac{1}{\sqrt{n !}} \hat{a}^{\star}\left(\phi_{1}\right) \cdots \hat{a}^{\star}\left(\phi_{n}\right)\right| 0>.
$$

The constant overall factor is a normalization constant introduced for convenience. The vacuum $\mid 0>$ is characterized as the only state that is destroyed by all annihilation operators and has unit norm. It corresponds to the vector $\phi_{s}^{(0)}=1$.

For any vector $f$ in $\mathcal{H}$, let us next introduce the Segal field operator $\hat{Y}_{R}$ on $\mathcal{F}_{0}$ [20], defined by

$$
\hat{Y}_{R}[f]=\frac{1}{\sqrt{2}}\left(\hat{a}(f)+\hat{a}^{\star}(f)\right) .
$$

In particular, the operator $\hat{Y}_{R}\left[f\left(u \mid u_{0}\right)\right]$ represents the bilocal field (5.1). The Segal field operator on $\mathcal{F}_{0}$ satisfies the commutation relations

$$
\left[\hat{Y}_{R}[f], \hat{Y}_{R}[g]\right]=i \operatorname{Im}<g, f>
$$

where Im denotes the imaginary part and we have set $\hbar=1$. This operator has a self-adjoint closure 20, which we will also call $\hat{Y}_{R}$. The spectral theorem [22] ensures then that $\exp \left(A \hat{Y}_{R}[f]\right)$ is a well-defined, self-adjoint, and positive operator for all vectors $f \in \mathcal{H}$, where $A$ is any real $c$-number.

Therefore, after replacing the field $Y(u)$ with its bilocal version $Y_{R}\left(u \mid u_{0}\right)$, we can represent the diagonal components $h_{a a}(u)(a=1$ or 2$)$ for the plane-wave metrics 2.3 by the regularized, positive operators:

$$
\begin{aligned}
\hat{h}_{a a}^{R}\left(u \mid u_{0}\right) & =\exp z_{0}(u) \exp \left\{-e^{-z_{0}(u)}\left\|f\left(u \mid u_{0}\right)\right\|^{2} / 2\right\} \\
& \times \exp \left\{(-1)^{a} \sqrt{2} e^{-z_{0}(u) / 2} \hat{Y}_{R}\left[f\left(u \mid u_{0}\right)\right]\right\} .
\end{aligned}
$$

Here, $\|f\|$ is the norm of $f \in \mathcal{H}$ and we have displayed the dependence on the points $u$ and $u_{0}$. We recall that, for the discrete model discussed in Sec. III, the points $u$ and 
$u_{0}$ have been restricted to lie in the interval $I_{L-\epsilon}$, whilst for the general case of linearly polarized waves analyzed in Sec. IV, $u$ and $u_{0}$ can take any real value. The second factor on the r.h.s. of this expression can be understood as follows. We choose to normal order the exponential of $\hat{Y}_{R}\left[f\left(u \mid u_{0}\right)\right]$, so that the vacuum expectation value of the operators (5.7) reproduces the classical value of the two-metric $h_{a b}$ when the field $Y$ vanishes, i.e., in flat spacetime. Employing then that (on $\mathcal{F}_{0}$ )

$$
\left[\hat{a}(f), \hat{a}^{\star}(g)\right]=<g, f>
$$

and the Campbell-Baker-Hausdorff (CBH from now on) formula $e^{\hat{b}} e^{\hat{c}}=e^{[\hat{b}, \hat{c}] / 2} e^{(\hat{b}+\hat{c})}$, valid for operators $\hat{b}$ and $\hat{c}$ whose commutator is a $c$-number [23], we finally arrive at the above expression for $\hat{h}_{a a}^{R}$.

We will not analyze in detail the introduction of an operator that represents the other independent component of the metric 2.3), which (after the change of coordinates $V=2 t-u$ ) can be written in the form $h_{u u}=z_{0}^{\prime}(u) e^{z_{0}(u) / 2} e^{\Phi(u)}$, the function $\Phi(u)$ being given by Eq. (2.4). As we will see in the next section, the discussion about the existence of large quantum effects that invalidate the classical description of the metric can be carried out without relying on a particular definition for this operator. Let us simply comment that

$$
\hat{\Phi}_{R}\left(u \mid u_{0}\right)=\int_{u_{c}}^{u} \frac{d r}{z_{0}^{\prime}(r)}:\left[\left(e^{-z_{0}(r) / 2} \hat{Y}_{R}\left[f\left(r \mid u_{0}\right)\right]\right)^{\prime}\right]^{2}:
$$

can be proved to be a densely defined operator on the symmetric Fock space $\mathcal{F}_{s}(\mathcal{H})$. Therefore, if $\hat{\Phi}_{R}\left(u \mid u_{0}\right)$ is essentially self-adjoint, its exponential [multiplied by the function $\left.z_{0}^{\prime}(u) e^{z_{0}(u) / 2}\right]$ provides a well-defined, positive operator that could be interpreted as a quantum counterpart of $h_{u u}$. Notice that $\Phi_{R}\left(u \mid u_{0}\right)$ has been obtained from the expression of $\Phi(u)$ by replacing the classical field $Y(u)$ with the Segal field operator $\hat{Y}_{R}\left[f\left(u \mid u_{0}\right)\right]$ and taking normal ordering.

\section{METRIC FLUCTUATIONS}

In this section, we will discuss whether there exist quantum states in our model that admit a classical description of the spacetime. It seems natural to consider as candidates the coherent states [23] of the basic field operator $\hat{Y}_{R}[f]$, since all these states are strongly peaked around classical field solutions. Actually, most of the analysis of large quantum gravity effects presented in the literature has been carried out by investigating the geometry fluctuations on states of this type [1, 12, 15]. In the models that have been considered, however, the geometry studied was obtained by a Killing reduction to three-dimensions. Here, we will study the quantum behavior of linearly polarized plane waves from a purely four-dimensional point of view. We are particularly interested in discussing the metric fluctuations on the vacuum, which is the coherent state that should represent flat spacetime.

Given any vector $c$ in the Hilbert space $\mathcal{H}$ (where $\mathcal{H}$ is $l^{2}$ for the model of Sec. III and $L^{2}\left(\mathbb{R}^{+}\right)$in the general case of linearly polarized waves), we define the coherent state $\mid c>$ by

$$
\left|c>=\exp \left(-\|c\|^{2} / 2\right) \exp \hat{a}^{\star}(c)\right| 0>.
$$

The overall numerical factor guarantees that $\mid c>$ has unit norm. Using formula (5.8) and recalling that the vacuum is destroyed by all annihilation operators, it is not difficult to see that, for any $f \in \mathcal{H}$, the expectation value of the Segal field on any coherent state is

$$
<\hat{Y}_{R}[f]>_{c}=\frac{1}{\sqrt{2}}(\langle c, f>+<f, c>) .
$$

Regardless of the value of $f$, this expectation value coincides with the classical field obtained by replacing the annihilation variables $a$ with the vector $c \in \mathcal{H}$ (and $a^{\star}$ with its complex conjugate, $\left.c^{\star}\right)$. On the other hand, for every fixed vector $f \in \mathcal{H}$, let us call $\hat{Y}_{R}[i f]$ the canonical momentum of the field $\hat{Y}_{R}[f]$. Notice that, from Eq. (5.6), the commutator of these two operators is the imaginary $c$-number $i\|f\|^{2}$. One can then check that all coherent states have the same uncertainty in the field $\hat{Y}_{R}[f]$ and in its canonical momentum. Furthermore, for all vectors $f \in \mathcal{H}$, the product of these uncertainties attains its minimum value [24], namely, $\|f\|^{2} / 2$.

Let us now analyze the quantum fluctuations in the metric. We will restrict our considerations to the operators (5.7), which describe the metric on the Killing orbits of our midisuperspace model. Our conclusions will be independent of the quantum behavior of $h_{u u}$, i.e., the other non-trivial component of the metric for plane waves with linear polarization. By defining

$$
F(u)=e^{z_{0}(u)}, \quad A_{a}(u)=(-1)^{a} \sqrt{2} F(u)^{-1 / 2}
$$

(with $a=1,2$ ) and suppressing from our notation the explicit dependence on the points $u$ and $u_{0}$, we can write Eq. (5.7) in the compact form

$$
\hat{h}_{a a}^{R}=F: \exp \left(A_{a} \hat{Y}_{R}[f]\right):,
$$

where the dots denote normal ordering. Employing the $\mathrm{CBH}$ formula and Eq. (5.8), one finds that

$$
<F: \exp \left(A_{a} \hat{Y}_{R}[f]\right):>_{c}=F \exp \left(\sqrt{2} A_{a} \operatorname{Re}<c, f>\right) .
$$

Here, we have used that $A_{a}$ is a real $c$-number, and $\mathrm{Re}$ stands for the real part. We therefore see that the expectation value of $\hat{h}_{a a}^{R}$ on a coherent state reproduces the classical value obtained from the field solution (6.2). In 
particular, for the vacuum state one gets the flat spacetime value $<\hat{h}_{a q}^{R}>_{0}=F \delta_{a}^{a}$.

From Eq. (6.5), it is straightforward to conclude that

$$
\left(\frac{\Delta_{c} \hat{h}_{a a}^{R}}{<\hat{h}_{a a}^{R}>_{c}}\right)^{2}=\exp \left(\frac{A_{a}^{2}\|f\|^{2}}{2}\right)-1 .
$$

For any observable $\hat{b}$, the symbol $\Delta_{c} \hat{b}$ denotes the uncertainty of the state $\mid c>$, namely, the square root of the difference between $\left.<\hat{b}^{2}\right\rangle_{c}$ and $\left(<\hat{b}>_{c}\right)^{2}$. It is worth remarking that, from the above expression, the relative fluctuations in the two-metric $\hat{h}_{a a}^{R}$ turn out to be independent of the particular coherent state considered. Note also that there will generally exist large fluctuations in the geometry whenever the expectation value of the metric $\hat{h}_{a a}^{R}$ is large. However, approximating this metric by the classical value (6.5) will still be acceptable provided that the relative fluctuations in $\hat{h}_{a a}^{R}$ are small. According to Eqs. (6.3) and (6.6), this will be the case if and only if

$$
e^{-z_{0}(u)}|| f\left(u \mid u_{0}\right) \|^{2} \ll 1 .
$$

Before continuing our discussion, we would like to comment on the implications that the uncertainty principle may have for operators of the form $\hat{h}_{a a}^{R}$, which are given by the normal ordered exponential of a Segal field multiplied by a positive $c$-number. Remember that $\hat{h}_{a a}^{R}$ denotes a different operator for each pair of points $u$ and $u_{0}$. Let then $\hat{b}=F: e^{\hat{Y}_{R}[f]}$ : and $\hat{c}=G: e^{\hat{Y}_{R}[g]}$ : be two such operators. We know that the uncertainty product in $\hat{b}$ and $\hat{c}$ is always greater than half the norm of the expectation value of $[\hat{b}, \hat{c}]$ [24]. In our case, the commutator $[\hat{b}, \hat{c}]$ is a quantum operator proportional to $F G: e^{\hat{Y}_{R}[f+g]}$ : Hence, its expectation value will depend on the state analyzed. As a consequence, it is not clear which is the minimum value allowed for the product of uncertainties in $\hat{b}$ and $\hat{c}$. In this situation, it seems more natural to consider, e.g., the uncertainty product divided by the expectation value of $F G: e^{\hat{Y}_{R}[f+g]}$ : This quantity turns out to be bounded by the $c$-number $\left|e^{<g, f>/ 2}-e^{<f, g>) / 2}\right| / 2$ and, on coherent states, coincides with the product of the relative fluctuations in $\hat{b}$ and $\hat{c}$, as can be checked by employing Eq. (6.5). Using techniques explained in Ref. 24, one can then prove that the considered quantity is not minimized by the coherent states for generic vectors $f, g \in \mathcal{H}$.

Let us now return to the analysis of the metric fluctuations. For the whole family of coherent states, we have seen that the classical description of the spacetime may be acceptable only if condition (6.7) is satisfied at all points $u$. Recall that $u_{0}$ is a conveniently chosen point where the diagonal components of the metric in the $x^{a}$ directions $(a=1,2)$ are set equal to $e^{z_{0}\left(u_{0}\right)}$ by convention, both classical and quantum mechanically. For the discrete model of Sec. III, the vector $f\left(u \mid u_{0}\right)$ denotes the sequence (3.6) in $l^{2}$, and for the model of Sec. IV, $f\left(u \mid u_{0}\right)$ is the function (4.6), which belongs to $L^{2}\left(\mathbb{R}^{+}\right)$. In general, inequality (6.7) will not be satisfied if the norm of the vector $f\left(u \mid u_{0}\right)$ or the function $e^{-z_{0}(u)}$ becomes considerably large at a certain point $u$. Since the form of $f\left(u \mid u_{0}\right)$ (and hence its norm) may depend on the regularization adopted in the quantization, we will concentrate our discussion on the possibility that $e^{-z_{0}(u)}$ is significantly large, a possibility that is insensitive to the ambiguities found in the construction of the quantum theory. Given the definition of $z_{0}$ in Eq. (2.4), the function $e^{-z_{0}(u)}$ is the double exponential of $-u$. This exponential becomes unbounded from above when $u$ approaches $-\infty$, which is precisely the region where null cones are focused by the plane wave, as we commented in Sec. II. We hence expect the quantum spacetime to radically differ from its classical approximation close to this focusing region.

In order to prove this statement, we only have to show that the norm of $f\left(u \mid u_{0}\right)$ remains strictly positive when $u \rightarrow-\infty$. It then follows that $\left\|f\left(u \mid u_{0}\right)\right\|^{-2}$ is bounded as a function of $u$ away from $u_{0}$ because, for every possible choice of the smearing function $g$ employed in our regularization, the vector $f\left(u \mid u_{0}\right)$ is normalizable and different from zero at all finite points $u \neq u_{0}$. As a consequence, condition (6.7) will not be satisfied when $u$ becomes large and negative 25].

Let us first analyze the model with discrete modes. We set $u_{0}=0$ and $u=-L / 3$ for convenience. In particular, if $L \gg \epsilon$, the points $u$ and $u_{0}$ belong to the interval $[-L+\epsilon, L-\epsilon]$ (to which we restricted our considerations in Sec. III). Using expression (3.6), it is not difficult to check that $\|f(-L / 3 \mid 0)\|>|\tilde{g}(\pi / L)|$. Since $\tilde{g}$ is the Fourier transform of a smooth function with unit integral, we have that $\tilde{g}(0)=1 / \sqrt{2 \pi}$. In the limit $L \rightarrow \infty$, when the point $u=-L / 3$ approaches the region where null geodesics are focused, the norm of $f(-L / 3 \mid 0)$ is then bounded from below by $1 / \sqrt{2 \pi}$. Therefore, in the case with discrete modes studied in Sec. III and assuming that the value of $L$ is sufficiently large, the relative fluctuations in the metric turn out to be huge when $u \ll 0$, regardless of the specific form taken by the function $\tilde{g}$. Thus, the classical spacetime breaks down for all coherent states in the region $0 \gg u>-L+\epsilon$.

In the general model of plane waves with linear polarization, one can also show that, in the limit $u \rightarrow-\infty$, the norm of the function $f\left(u \mid u_{0}\right)$ is bounded from below by a strictly positive number [25,26] for every possible choice of the function $g$ used in the regularization. Hence, we conclude again that the relative uncertainty in the metric $\hat{h}_{a a}^{R}$ is large close to the hypersurface where null cones are focused, i.e, for large and negative values of the coordinate $u$. In that region at least, the classical description of the spacetime, as a plane wave with $x^{a}$-components of the classical metric given by Eq. (6.5), is not acceptable for any of the coherent states. Notice that this breakdown of the classical spacetime when one approaches the fo- 
cusing region is regulator independent, because it occurs for all of the admissible choices of smearing function $g$. Finally, let us emphasize that our results apply as well to the vacuum, which is the quantum state that should describe flat spacetime. So, quantum gravitational effects around the vacuum cannot be neglected in the vicinity of the region where null geodesics are focused by linearly polarized plane waves.

\section{SUMMARY AND DISCUSSION}

Linearly polarized plane waves in source-free gravity have been described by a midisuperspace model that is free of constraints and whose reduced Hamiltonian vanishes. This model was obtained in Ref. [3], starting with the Hamiltonian formulation of general relativity for spacetimes with two commuting spacelike Killing vectors and introducing gauge-fixing and symmetry conditions. The degrees of freedom of the model are given by a field $Y$ that (on classical solutions) depends only on one of the spacetime coordinates, namely, the coordinate $u$. We have shown that one can consistently restrict all considerations to fields that vanish at a given point $u_{0}$. Furthermore, after a Fourier expansion, this field can be expressed in terms of an infinite collection of annihilation and creation like variables. In the general case of linearly polarized waves, these variables form a continuous set, but we have also considered a simplified version of the model in which the field $Y$ describes flat spacetime outside a fixed region. If one is only interested in studying that region, the Fourier expansion of the field can be restricted to a discrete set of modes. Finally, we have argued that the position of the points $u$ and $u_{0}$ cannot be determined with total accuracy in any physical measurement. As a consequence, the field measured, $Y_{R}\left(u \mid u_{0}\right)$, would result from an average over some small neighborhood of those points.

In order to quantize the system, we have introduced a Fock representation and described the field $Y_{R}\left(u \mid u_{0}\right)$ by a Segal field operator. This Segal quantization has been carried out over the Hilbert space of square summable sequences for the model with discrete modes, and over the space of square integrable functions on the positive real line for the general case of plane waves with linear polarization. In this way, we have been able to construct regularized operators that represent the spacetime metric for plane waves. At least for the two-metric on Killing orbits (i.e., the sections of constant coordinates $t$ and $u$ ), we have proved that these regularized operators are self-adjoint and positive. We have then concentrated our discussion on the analysis of the coherent states of the quantum theory. These states are characterized by minimizing the uncertainty product in any Segal field and its conjugate momentum. We have checked that the expectation value of the metric (on Killing orbits) reproduces in fact the classical solution that one would obtain from the expectation value of the Segal field. However, the relative fluctuations in this metric become huge for all coherent states when the coordinate $u$ gets large and negative. Such values of $u$ describe the vicinity of a region where null cones are focused by the plane wave 16$]$. Therefore, the quantum geometries represented by coherent states do not admit an approximate classical description in that region and classical spacetime breaks down.

It is worth remarking that this result applies as well to the vacuum of the model, which should correspond to flat spacetime. In this sense, the spacetime foam around the vacuum turns out to be quite significant, at least close to the focusing region. This fact casts serious doubts on the possibility that, after introducing matter fields in the system, a quantum field theory around the flat-spacetime solution of our model could provide a good semiclassical approximation [11] to the full quantum theory.

It would be interesting to analyze the metric fluctuations for families of quantum states other than coherent states. Obviously, this analysis would not affect our conclusions about the vacuum. In addition, note that, even if it were possible to find states with smaller metric uncertainties, this would be done at the cost of losing coherence in the Segal field.

On the other hand, the results attained might well depend on several choices that have been made either in the construction of the model or in the quantization process. Among such choices, let us briefly comment on the representation selected for the quantum theory, on the mode decomposition and regularization adopted for the basic field $Y$, and on the system of coordinates employed to describe the plane waves.

The commutation relations (5.8) admit representations other than that discussed in Sec. V. For instance, one could have made a different choice of creation and annihilation operators that led to a unitarily equivalent Fock representation but with a different vacuum. In this sense, the question that is physically important is the correct identification of the state represented by the vacuum in our quantum theory. This vacuum is the only normalized state with minimum uncertainty in the Segal field $\hat{Y}_{R}[f]$ and its canonical momentum (for all vectors $f$ ) that is peaked around the zero field. Since spacetime is flat when the classical field $Y$ vanishes, it seems reasonable to identify our vacuum as the state corresponding to the flat solution in the constructed quantum theory.

One can also find representations of our commutation relations that are unitarily inequivalent to that employed in Sec. V. It then could happen that our conclusions were not valid for some of such representations. It is worth noticing, nevertheless, that the Fock representation adopted guarantees that the Segal field $\hat{Y}_{R}[f]$ (i.e., the basic field of the theory) is a self-adjoint operator for all vectors $f$. In addition, the existence of a cyclic 20 vacuum that describes the classical flat solution obtained when the field $Y$ vanishes makes of our choice of representation a natural selection. In any case, the aim of this work is not at showing that a classical description 
of the spacetime is precluded in quantum gravity for all possible representations, but that the existence of such an approximate description cannot be taken for granted until the quantum theory is known.

On the other hand, in terms of the coordinate $u$, the expansion in modes employed for our basic field $Y(u)$ is most natural, since it is its standard Fourier expansion. Nevertheless, one might have chosen to expand the field in the Fourier modes associated with another coordinate $\bar{u} \in \mathbb{R}$, related to $u$ by means of a (differentiable) bijective transformation $u=H(\bar{u})$ [so that $H^{\prime}(\bar{u}) \neq 0$ ]. One can always set $H^{\prime}(\bar{u})$ to be positive, e.g., by using the invariance of the geometry under a reversal of the null coordinates $V$ and $\bar{u}$. In addition, the considered change of coordinate amounts in fact to a different selection of function $z_{0}$ in Eq. (2.4), namely, $\bar{z}_{0}(u) \equiv z_{0}[H(u)]$. Remarkably, it turns out that the gauge fixing and symmetry reduction carried out in Ref. [3], as well as the analysis and quantization performed in the present work [except for definition (2.2)], continue to be valid with the replacement of $z_{0}(u)$ with $\bar{z}_{0}(u)$, provided that the latter is a strictly increasing function that ranges over the whole negative axis. One can easily see that the function $\bar{z}_{0}(u)$ analyzed here satisfies these conditions. As a consequence, adopting a Fourier expansion in terms of the coordinate $\bar{u}$ can be considered equivalent to the introduction of a different gauge fixing for the $u$-coordinate diffeomorphisms, and leads just to an alternative representation of our commutation relations that is carried by a (possibly) different Fock space. Actually, it is not difficult to show that such a representation is related to that introduced in Sec. $\mathrm{V}$ by a Bogoliubov transformation of the creation and annihilation operators. Moreover, one can check that all the coherent states of the quantum theory that is obtained with this alternative expansion in modes present also large relative fluctuations in the metric when one approaches the focusing region.

As for the regularization of our basic field, we recall that the infrared divergences have been eliminated by imposing that the field vanishes at $u_{0}$, both classical and quantum mechanically, whereas the ultraviolet divergences have been removed by averaging the field around the points $u$ and $u_{0}$ with a smooth function $g$ of compact support, an average that amounts to a smearing of the field. The choice of the point $u_{0}$ can be made on physical grounds: the value of the field at that point is taken as a reference value for all measurements. On the other hand, it seems reasonable to assume that the support of the function $g$ is of the order of the Planck length. The form of this function, however, remains quite arbitrary. This introduces an ambiguity in the quantization process, which is similar to that encountered in Ref. [1] when defining the regularized metric for (the dimensional reduction of) cylindrical waves with linear polarization. The conclusion that the metric fluctuations become large for coherent states when one approaches the region where null cones are focused is nevertheless regularization independent, as we argued in Sec. VI, and is basically due to the fact that the metric on the two-dimensional Killing orbits degenerates in the focusing region [so that $e^{z_{0}}$ vanishes in Eq. (6.7)].

Our last comments refer to the system of coordinates employed. This system does not cover the totality of the spacetime that can be described with harmonic coordinates. Moreover, the region where the metric uncertainty explodes $(u=-\infty)$ corresponds to a coordinate singularity in group coordinates, and this singularity can be removed by introducing harmonic coordinates [8]. One might then wonder whether the huge quantum fluctuations detected could have been avoided by adopting harmonic coordinates.

We remind, nonetheless, that the spacetimes described by those coordinates are not globally hyperbolic 16 . Since the canonical formulation of general relativity can only be applied to spacetimes which can be foliated in time slices, the quantization of gravitational plane waves using harmonic coordinates cannot be achieved by standard methods. Our results will be relevant for the whole of the spacetime covered with a single chart in harmonic coordinates provided that one can neglect the effect of the regions that are not described by our system of coordinates. One expects this to be the case, at least, for sandwich waves that leave the spacetime flat in those regions. More importantly, one might have expected that the quantization process would actually smooth out the strong gravitational effects exerted by the plane wave in the region where null cones are focused (i.e., in the vicinity of the coordinate singularity in group coordinates), in a similar way as it is usually expected that spacetime singularities disappear in quantum gravity. On the contrary, and at least for the spacetimes described by coherent states, our results prove that such strong effects are in fact enlarged in the quantum theory.

\section{ACKNOWLEDGMENTS}

The authors are grateful to P. F. González-Díaz for valuable discussions and comments. They are also thankful to A. Roura and E. Verdaguer for discussions. G. A. M. M. acknowledges DGESIC for financial support under the Research Projects No. PB97-1218 and No. HP19880040. M. M. was supported by CICYT under the Research Project No. AEN98-04031 and by funds provided by a Basque Government FPI grant.

[1] A. Ashtekar and M. Pierri, J. Math. Phys. 37, 6250 (1996).

[2] D. Korotkin and H. Samtleben, Phys. Rev. Lett. 80, 14 (1998). 
[3] G. A. Mena Marugán and M. Montejo, Phys. Rev. D 58, 104017 (1998).

[4] G. A. Mena Marugán, Phys. Rev. D 56, 908 (1997).

[5] K. Kuchař, Phys. Rev. D 4, 955 (1971); M. Allen, Class. Quantum Grav. 4, 149 (1987).

[6] D. E. Neville, Class. Quantum Grav. 10, 2223 (1993); Phys. Rev. D 55, 766 (1997); 55, 2069 (1997); 56, 3485 (1997); 57, 986 (1998); R. Borissov, ibid. 49, 923 (1994).

[7] See also B. K. Berger, Ann. Phys. (N.Y.) 83, 458 (1974); 156, 155 (1984); V. Husain and L. Smolin, Nucl. Phys. B327, 205 (1989).

[8] E. Verdaguer, Phys. Rep. 229, 1 (1993).

[9] R. H. Gowdy, Ann. Phys. (N.Y.) 83, 458 (1974); 156, 155 (1984).

[10] See, for instance, A. Ashtekar, R. S. Tate, and C. Uggla, Int. J. Mod. Phys. D 2, 15 (1993); H. Kastrup and T. Thiemann, Nucl. Phys. B399, 211 (1993); B425, 665 (1994); N. Manojlović and G. A. Mena Marugán, Phys. Rev. D 48, 3704 (1993); G. A. Mena Marugán, ibid. 53, 3156 (1996).

[11] R. Wald, Quantum Field Theory in Curved Space-Times and Black Hole Thermodynamics (University of Chicago Press, Chicago, 1994).

[12] A. Ashtekar, Phys. Rev. Lett. 77, 4864 (1996).

[13] A. E. Domínguez and M. H. Tiglio, gr-qc/9905086.

[14] R. Gambini and J. Pullin, Mod. Phys. Lett. A 12, 2407 (1997).

[15] C. Beetle, Adv. Theor. Math. Phys. 2, 471 (1998).

[16] R. Penrose, Rev. Mod. Phys. 37, 215 (1965).

[17] H. Bondi, F. A. E. Pirani, and I. Robinson, Proc. R. Soc. London A251, 519 (1959).

[18] D. Kramer, H. Stephani, M. MacCallum, and E. Herlt, Exact Solutions of Einstein's Field Equations (Cambridge University Press, Cambridge, England, 1980).

[19] D. R. Baldwin and G. B. Jeffrey, Proc. R. Soc. London A111, 95 (1926).

[20] M. Reed and B. Simon, Methods of Modern Mathematical Physics II: Fourier Analysis, Self-Adjointness (Academic Press, New York, 1975).

[21] J. Tarski, J. Math. Phys. 5, 1713 (1964); G. Morchio and F. Strocchi, Ann. Inst. H. Poincaré A 33, 251 (1980); G. Morchio, D. Pierotti, and F. Strocchi, J. Math. Phys. 31, 1467 (1990); E. Abdalla, M. C. B. Abdalla, and K. D. Rothe, Two Dimensional Quantum Field Theory (World Scientific, Singapore, 1991).

[22] See, for instance, M. Reed and B. Simon, Methods of Modern Mathematical Physics I: Functional Analysis (Academic Press, New York, 1972).

[23] See, for instance, L. Mandel and E. Wolf, Optical Coherence and Quantum Optics (Cambridge University Press, Cambridge, England, 1995).

[24] R. Jackiw, J. Math. Phys. 9, 339 (1968).

[25] We assume that the choice of $u_{0}$ is such that the distance $\left|u-u_{0}\right|$ is bounded from below by a strictly positive constant for $u \ll 0$.

[26] Actually, the norm of $f\left(u \mid u_{0}\right)$ generally diverges when $|u| \rightarrow \infty$. A case in which the norm remains finite in that limit is when one considers that what is kept fixed is the distance $\left|u-u_{0}\right|$, rather than the location of the reference point $u_{0}$. 